\title{
Is It an Ovarian Tumor or Broad Ligament Leiomyoma?
}

\author{
Jihad ALHASSAN ${ }^{1}$, Mayssaloun KHAIRALLAH ${ }^{2}$, Kariman GHAZAL ${ }^{2 *}$, Zaynab HAROUN² and Eva KOULAYMI² \\ ${ }^{1}$ Head of department of obstetrics and gynecology, AL ZAHRAA University Hospital, Lebanon
}

${ }^{2}$ Department of obstetrics and gynecology, AL ZAHRAA University Hospital, Lebanon

*Corresponding author: Kariman GHAZAL, Department of obstetrics and gynecology, AL ZAHRAA University Hospital, Lebanon.

Received Date: December 02, 2020

Published Date: January 05, 2021

\begin{abstract}
Uterine leiomyomas, also known as myomas or fibroids, are by far the commonest benign uterine tumors. Leiomyomas are not uncommon in the round, ovarian and broad ligaments. They can be misdiagnosed as ovarian tumor even by MRI that can give typical findings of ovarian neoplasm, thereby altering the course of treatment offered.

A menopaused 53 years old lady, biparous, presented to our clinic for regular check-up. She is previously healthy and denies any complain. She has however a family history of ovarian cancer. General physical examination was unremarkable. The vagina and cervix appeared healthy on gross visualization without any discharge or vaginal bleed.

Ultrasonography of pelvis revealed a well-defined heterogeneous right adnexal mass of $4.3 x 3.26 \mathrm{~cm}$. MRI showed a $38 x 39 \mathrm{~mm}$ isointense in both T1 and T2 right adnexal mass with an impression of fibro-thecoma. Serum Ca -125 (cancer antigen) $31 \mathrm{U} \backslash \mathrm{mL}$.

The family history as well as the aspect of the mass on ultrasound and MRI raised the suspicion of an ovarian tumor. However, Laparoscopy revealed a mass arising from the right broad ligament whose Histopathological examination confirmed a broad ligament leiomyoma that was removed laparoscopically.

Laparoscopic approach for broad ligament fibroid can achieve good results. Till present, there is very few data on the ease of imaging of broad ligament leiomyoma as it would not help ruling out the origin of adnexal mass accurately.
\end{abstract}

Keywords: Broad ligament leiomyoma; Laparoscopy; Pelvic ultrasound

\section{Introduction}

Leiomyoma is a smooth muscle tumor that turns into cancer in $0.1 \%$ of the cases [1]. It occurs in 20_30\% of women older than 35 years old and represents the most common uterine neoplasm [1]. Uncommonly, it can be found in extra uterine locations such as the broad ligament, cervix or it can exist as diffuse leiomyomatosis [2]. Despite that broad ligament is the most common site, it exists only in less than $1 \%$ of cases $[2,3]$. It is divided into 2 types:

a) False broad ligament tumor: which is a uterine tumor expanding to the broad ligament. b) True or primary broad ligament tumor arising from the sub peritoneal connective tissue of the ligament [4].

While in most cases broad ligament leiomyomas are asymptomatic, patients may present with pelvic pain or a palpable mass as a result of pressure effects on adjacent organs [5].

Therefore, in most of the cases imaging such as ultrasound, CT scan, and MRI are the main diagnostic tools.

Here we present a case of broad ligament leiomyoma misdiagnosed as ovarian mass, managed by laparoscopy. 
A 53 years old lady, biparous, with previous 1 normal delivery and 1 cesarean section, menopaused 1 year ago, presented to our clinic for regular annual check-up. While interrogating her, she denied history of pelvic pain, anorexia, weight loss or any bowel or bladder complaints. She mentioned that her mum and sister both died from ovarian cancer. She is not febrile, and vitals were within normal range. While examining her abdomen, it was soft with no palpable mass or abdominal or pelvic tenderness upon palpating her.

On speculum examination, before pap smear swab, the vagina appeared healthy on gross visualization and cervix looked atrophic with mild deviation to left side. There was no discharge or vaginal bleeding. Because of a strong family history of ovarian cancer, a check-up ultrasound showed a well-defined heterogeneous right adnexal mass of $4.3 \times 3.26 \mathrm{~cm}$. A decision for further workup on a post-menopaused lady with an ovarian mass was taken. MRI showed a $38 \times 39 \mathrm{~mm}$ isointense in both T1 and T2 right adnexal mass with an impression of fibro-thecoma. Serum Ca -125 (cancer antigen) turned out to be $31 \mathrm{U} \backslash \mathrm{mL}$.

The family history as well as the aspect of the mass on ultrasound and MRI raised the suspicion of an ovarian tumor.

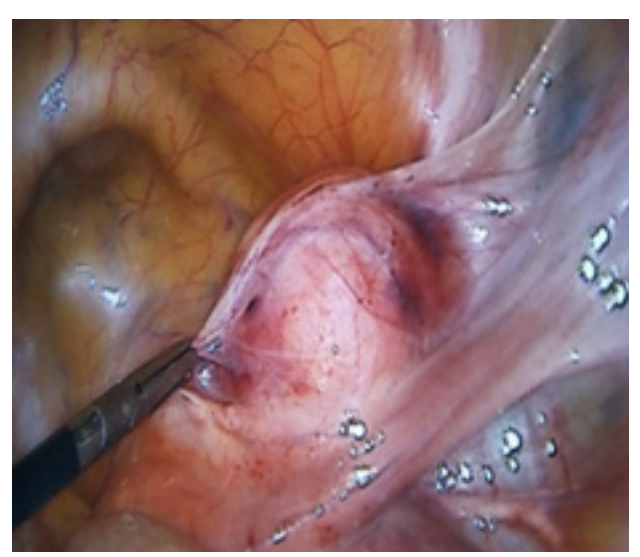

Figure 1: Right broad ligament fibroma.

Tumor and the decision for exploratory laparoscopy with possible right oophorectomy with frozen section was taken. A 10 mm supra-umbilical incision was made for the camera, in addition to two lower quadrants, above and medial to the ASIS, taking care of to avoid the inferior epigastric artery, incision for a $5 \mathrm{~mm}$ and $10 \mathrm{~mm}$ trocars respectively. Intra-operatively, a well circumscribed right broad ligament mass was identified (Figure 1). The uterus, the ovaries and fallopian tubes had normal morphologies. We started by opening the Broad ligament using a bipolar, an incision was made in the anterior aspect of the fibroid (Figure 2) that was stripped off the peritoneum carefully until reaching the base that was cut using a ligature. The course of the ureter was traced at the beginning and at the end of the procedure. Good homeostasis was ensured (Figure 3). The operation course was smooth without any complications, with an estimated blood loss of 100 cc. Fibroma was removed from the abdomen by extending the RLQ incision, after placing it in an endo-bag. The broad ligament was sutured using biosyn 2-0 (Figure 4). Specimen was sent for histopathological examination that revealed typical leiomyoma tissue description: thin spindle cells in a whorled arrangement (Figure 5).

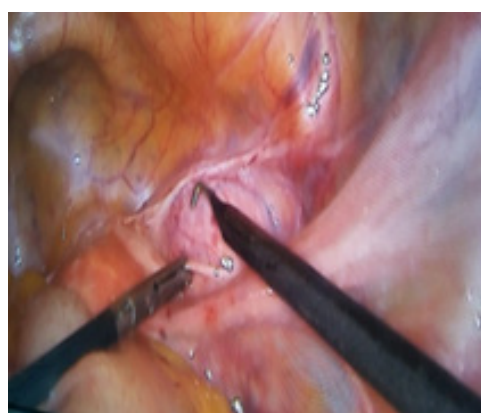

Figure 2: Incision of the anterior aspect of the fibroid.

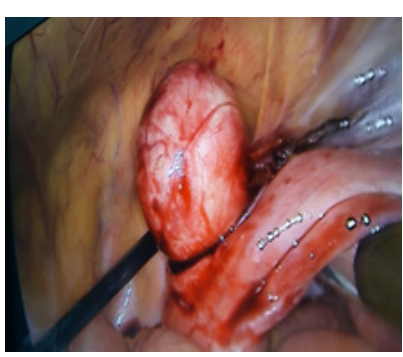

Figure 3: Peeling of the myoma until reaching the base.

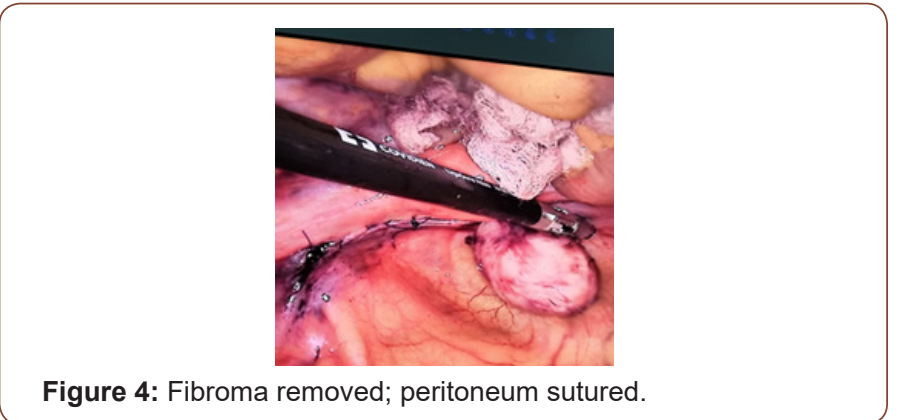

Figure 4: Fibroma removed; peritoneum sutured.

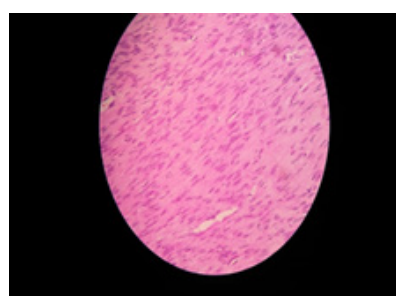

Figure 4: Microscopic view of the broad ligament fibroid. Note the spindle cells in a whorled arrangement.

Patient was clinically and hemodynamically stable post op. She had a smooth hospitalization course and was discharged home in the following day in good clinical conditions.

\section{Discussion}

leiomyomas are non-cancerous growth of smooth muscles [6]. Although uncommon, extra uterine leiomyomas can occur $[5,6]$. 
They develop an auxiliary blood supply and lose their original attachment at the uterus [6]. Broad ligament leiomyoma occurs in less than $1 \%$ of cases [7]. Some authors suggest that broad ligament leiomyomas originate from hormonally sensitive smooth muscle elements of it [5]. In fact, broad ligament leiomyoma can be asymptomatic but can also cause mass effect symptoms, as well as, it can affect reproduction by causing infertility, miscarriage and premature labour [5,6]. Broad ligament leiomyoma is generally diagnosed by imaging. As ultrasound is least invasive and most cost effective, it is the preferred imaging modality for initial evaluation [6-8]. A leiomyoma has a typical pattern: a whorled appearance with variable echogenicity depending on the extent of degeneration, fibrosis and calcification [5-8]. Transvaginal ultrasound is superior to transabdominal ultrasound as it may help in a clear visualization of the ovaries and uterus from the mass [5]. Although easily recognized, leiomyomas have been misdiagnosed as adenomyosis, hematometra, uterine sarcoma, ovarian mass as in our case.

MRI can however define the anatomy of the uterus and ovaries, but this is limited by its availability and high cost [6]. On MRI, leiomyomas show typically hypointense signal on both T1 and T2 weighted images. Contrary to Rojanna, et al. who mentioned that MRI is the image of choice to differentiate broad ligament and masses of ovarian or tubal origin [5]. Nevertheless, this wasn't the case in our patient were MRI confirmed what ultrasound showed [5]. As per Kindiger, et al. accurate preoperative diagnosis was made only in 1 in 10 broad ligament leiomyomas, thus the challenge posed by broad ligament leiomyomas at preoperative imaging [8]. It may be confused with ovarian tumor by appearing adnexal in location [8]. This is very crucial as broad ligament leiomyomas are associated with pseudo-Meigs syndrome and produce high level of CA-125 pointing towards a metastatic ovarian carcinoma causing thereby diagnostic confusion [5]. Rojanna, et al. have described a case of a broad ligament leiomyoma mimicking an ovarian tumor on ultrasound and CT scan as well. In their case, however, the fibroid had cystic degeneration [5]. By then, an ultrasound guided percutaneous biopsy of the tumor might be helpful to determine its exact histologic composition. Intraoperative frozen section would be another option.

Myomectomy is generally indicated only in symptomatic case such as menorrhagia, pelvic pain, pressure symptoms and infertility [8]. Prandit, et al. mentioned that only 2 cases of true broad ligament leiomyoma were operated. With experienced hands, laparoscopy is the best route for broad ligament leiomyoma as patient has shorter hospital stay, less morbidity and faster recover as compared to abdominal approach $[8,9]$. There is paucity of literature on the laparoscopic management of leiomyoma within the broad ligament. Enucleation of broad ligament leiomyoma is difficult because of rich vascularity. Urethral injury, uterine vessel injuries and concealed hematoma formation are common complications [810]. Sakanaka, et al. have reported laparoscopic removal of 7 broad ligament leiomyomas with a safe operative course and encourage laparoscopic treatment of broad ligament leiomyomas [10]. Such operation should be attempted by most experienced gynecologist surgeons. Optimally, perioperative GnRH analogues can be used to reduce size and vascularity of the f leiomyoma. However, it might cause loss of cleavage planes [9]. Given the potential complications of broad ligament myomectomy, surgical anticipation is crucial particularly for the unexperienced hands. This may reflect the lack of awareness among radiologist of the surgical significance of broad ligament location. At present, there is very few data on the ease of imaging of broad ligament leiomyomas and their safe laparoscopic management. A careful perioperative care and appropriate surgical management are necessary to obtain satisfactory results.

\section{Conclusion}

Broad ligament leiomyoma is a rare entity, and a true Broad ligament, as in our case, is the rarest. Broad ligament leiomyomas, though benign, can be confused with ovarian tumor. This case will facilitate in creating a clinical awareness about the difficulty in its diagnosis and will encourage its management by laparoscopy, which, in our case, helped us in both diagnosis and treatment.

\section{Acknowledgement}

None.

\section{Conflict of Interest}

Authors declare no conflict of interest.

\section{References}

1. Fasih N, Prasad Shanbohogue AK, Macdonald DB, Fraser-Hill MA, Papadatos D, et al. (2008) Leiomyomas beyond the uterus: unusual locations, rare manifestation. Radiographics 28(7): 1931-1948.

2. Neha G, Manisha L (2014) A Rare Case of Giant Broad Ligament Fibroid with Cervical Fibroid Mimicking Ovarian Tumour. International Journal of Recent Trends in Science and Technology 10(2): 208-209.

3. Bhatta N (2001) Tumours of the Corpus Uteri. Jeffcoats Principles of Gynaecology. $6^{\text {th }}(\mathrm{edn})$, Arnold Printers, London, p.470.

4. Kumar P, Malhotra N (2008) Jeffcoate's Principles of Gynaecology. $7^{\text {th }}$ (edn), Jaypee Brothers, New Delhi, India, p.492.

5. Rajanna D, Pandey V, Janardhan S, Datti SN (2013) Broad ligament fibroid mimicking as ovarian tumor on ultrasonography and computed tomography scan. J Clin Imaging Sci 3: 8.

6. Aydin C, Eriș S, Yalçin Y, Selim HS (2013) A giant cystic leiomyoma mimicking an ovarian malignancy. Int J Surg Case report 4: 1010-1012.

7. Bakari F, Sulayman H, Avidime S, Ameh N, Adesiyun AG (2015) Huge broad ligament leiomyoma: a case report. Case reports in clinical medicine 4: 53833

8. Kindiger LM, Setchell TE, Miskry TS (2014) Broad ligament fibroids- a radiological and surgical challenge. Gynecol surg 11: 19-22.

9. Prandit P, Chandak S (2011) Laparoscopic management of broad ligament fibroids. J Gynecol Endosc Surg 2(1): 64-66.

10. Sakanaka M, Kohno K, Arai Y, Nishida M (2013) Management of seven cases of broad ligament fibroids via laparoscopic surgery. Japanese journal of gynecologic and obstetric endoscopy 29 (1): 79-83. 